\title{
Curvature effect on droplet impacting onto hydrophobic and superhydrophobic spheres
}

\author{
Danial Khojasteh a, Alireza Bordbar ${ }^{\text {a }}$, Reza Kamali ${ }^{\text {a,1 }}$ \& Marco Marengo ${ }^{\text {b }}$ \\ ${ }^{a}$ School of Mechanical Engineering, Shiraz University, Shiraz, 71936-16548, Iran \\ ${ }^{b}$ School of Computing, Engineering and Mathematics, University of Brighton, BN2 4GJ \\ Brighton, United Kingdom
}

danial.khojasteh@shirazu.ac.ir, alireza.bordbar@shirazu.ac.ir, rkamali@shirazu.ac.ir, m.marengo@brighton.ac.uk

D. Khojasteh, A. Bordbar, R. Kamali, M. Marengo

${ }^{1}$ Corresponding author 


\title{
Curvature effect on droplet impacting onto hydrophobic and superhydrophobic spheres
}

\begin{abstract}
Droplet impact on hydrophobic and superhydrophobic solid surfaces finds numerous applications, while the wide range of the parameters affecting its outcome necessitate a thorough study to reveal the underlying physics. Specific applications are related to the drop impact upon curved surfaces, such as microencapsulation in fluidized beds. Three-dimensional numerical simulations by applying Level-Set Method have been performed to investigate the water droplet impact on curved and flat hydrophobic and superhydrophobic substrates. Parameters such as the impact Weber number, the surface curvature and the equilibrium contact angle have been varied in order to assess their effects on the dynamics of the impact process. After providing a strong validation, it is found that impact on spherical surfaces generally presents a higher area of liquid to be in contact with the substrate with respect to the case of flat surfaces, when all other impact conditions are the same.
\end{abstract}

Keywords: droplet impact; curved surfaces; spherical surface;

superhydrophobicity; wettability.

\section{Introduction}

Over the past few decades, the dynamics of droplet impact on solid surfaces has become the subject of numerous studies due to its implications in a wide range of industrial applications, including spray cooling (Kim 2012; Binesh, Mousavi, and Kamali 2015), inkjet printing (Yusof et al. 2011; Kamali, Khojasteh, and Mousavi 2016), impingement of fuel droplets on the walls of combustion chambers (Liu et al. 2016), droplet-based microfluidic (Dehghan Manshadi et al. 2016a, 2016b), etc. In addition, some applications are specifically related to impact upon superhydrophobic (SH) surfaces; such as self-cleaning and anti-icing for lab-on-chip devices, and coating processes used in aerospace and power industries (Khojasteh et al. 2016b). Basically, in order to investigate surface wettability and study the droplet-surface interaction, two approaches - dynamic and static approach - are commonly adopted (Antonini, Amirfazli, 
and Marengo 2012). In static approach, sessile drops are employed, and in dynamic approach, pursued in the current study, the impact of droplets on solid surface are considered. Many factors are involved in altering the outcomes of drop impact on different surfaces including impact velocity, its direction relative to the surface, drop size, the properties of the liquid (its density, viscosity, viscoelasticity or other non-Newtonian effects), the surface or interfacial tension, the roughness and wettability of the solid surface, the non-isothermal effects (such as solidification and evaporation) and air entrapment (Yarin 2006), and extensive studies have been dedicated to impact phenomena on planar substrates, taking into account the aforementioned parameters (Tsai et al. 2009).

Wettability of the surface is commonly stated in terms of the contact angle (C.A) of a water droplet on it and a SH-surface is generally defined as one with a high C.A (usually stated to be $150^{\circ}$ and higher) and a low C.A hysteresis (lower than $10^{\circ}$ ). C.A hysteresis is the difference between the advancing and receding C.A.s and is often used as a measure of drop mobility on a surface (Antonini, Amirfazli, and Marengo 2012). On the other hand, a small C.A (less than $90^{\circ}$ ) leads to a reasonably well spread of water droplets on the surface, and hence these surfaces are referred to as hydrophilic (Xu and Heath 2013). The interval between these two ranges is assigned to hydrophobic $(\mathrm{H})$ surfaces (Khojasteh et al. 2016b). It has been observed that droplets experience two distinct phases upon impacting on $\mathrm{SH}$-surfaces, referred to as spreading and retracting (Liu et al. 2015). In the first phase, the droplet is flattened and its kinetic energy is converted to interfacial energy with a slight energy dissipation. During the second phase, the droplet tends to take on a spherical form again and eventually gets detached in vertical direction. Previous studies have shown that rebounding, in which the droplet finally bounces off the surface, occurs at low impact velocities (Aboud and Kietzig 2015) and 
above a critical maximum velocity, water remains on the surface and is not able to lift off (Antonini et al. 2013). This is called transition from Cassie-Baxter to Wenzel wetting state. In fact, Cassie-Baxter state explains the situation in which air is trapped between the droplet and the solid surface in a space provided by surface roughness, while in Wenzel state, this region is filled with liquid and air pockets are displaced.

Weber (We) and Reynolds (Re) numbers are among the main dimensionless numbers governing the impact phenomena which are defined as $W e=\frac{\rho V_{0}^{2} D}{\sigma}$ and $R e=$ $\frac{\rho V_{0} D}{\mu}$, respectively, where $\rho, \mu$, and $\sigma$ represent liquid's density, viscosity, and surface tension, respectively, $D$ denotes the drop's diameter and $V_{0}$ is the velocity of impingement.

Hartley and Brunskill were the first to study the effect of wettability on droplet rebound after impact onto leaves (Hartley and Brunskill 1958). Antonini et al. (1958) conducted an experimental study to clarify the conditions for drop rebound on $\mathrm{H}$ - and SH-surfaces. They observed that in addition to surface hydrophobicity, receding C.A is a key wetting parameter that affects drop rebound and determined a threshold receding C.A for which rebound occurs. Bange and Bhardwaj (2016), in a numerical study, investigated the bouncing and non-bouncing behavior of droplets during isothermal impact on $\mathrm{SH}$ surfaces. They found that the droplet bounces off the surface just in case the total energy of the droplet exceeds the sum of initial surface and gravitational energy at the instant of maximum recoiling. A brief review of potential applications of $\mathrm{SH}$-surfaces in addition to a thorough focus on the most recent advances regarding dynamics and kinematics of drop impinging SH substrates is provided by Khojasteh et al. (2016b). In other works (Khojasteh, Mousavi, and Kamali 2017; Khojasteh et al. 2016a), they analyzed the behaviors of Newtonian and shear-thinning non-Newtonian droplets impinging on heated 
HP- and H-surfaces by considering the effects of variations in We number, C.A and surface temperature.

While a large number of experimental studies, analytical modellings and numerical simulations have been dedicated to impact phenomena upon planar surfaces, very limited literature can be found considering impact on non-planar, i.e. curved surfaces, despite the fact that this phenomenon has a wide range of technological and industrial applications including micro-encapsulation in the fluidized beds, as well as drop impact onto structured, textured or rough surfaces. The results are useful also for better understanding of drop impact onto wires, cables or antennas - which is important for example in describing the icing effect and spray coatings. Another situation where this phenomenon can be observed is in Multi-Effect Desalination (MED) evaporators, where sea water is sprayed on a tube bundle carrying hot steam in order to obtain distilled water inside the tubes. Hung and Yao (1999) experimentally analyzed the drop impact on cylindrical wires and considered the effects of droplet velocity and wire size. They classified the post-impingement behaviors as either disintegrating at high impact velocities or clinging to the wire and eventually dripping off at low impact velocities. Hardalupas, Taylor, and Wilkins (1999) conducted experiments on liquid droplets with size and velocity ranges of 160 to $230 \mu \mathrm{m}$ and 6 to $13 \mathrm{~m} / \mathrm{s}$, respectively, impinging on the surface of a solid sphere with a diameter of about $1 \mathrm{~mm}$. Their results showed that the impinged droplet formed a crown which was influenced by surface roughness and kinematic and liquid properties of the droplet. Bakhshi, Roisman, and Tropea (2007) carried out experimental and theoretical investigations into the impact of a droplet onto a spherical target. They found three distinct temporal phases of the film dynamics from the experimental results, namely the initial drop deformation phase, the inertia dominated phase, and the viscosity dominated phase. 
Since all of the above-mentioned studies of impact on curved surfaces were mainly conducted experimentally, increasing attention is drawn towards numerical simulation of these phenomena. In this regard, Pasandideh-Fard, Bussmann, and Chandra (2001) developed a three-dimensional computational model to simulate the impact of water droplets with constant diameters on stainless steel tubes with variable diameters. They concluded that as the diameter of the tube is decreased, less liquid remained on the tube after impact, and most of it fell off in the form an unstable liquid column. Liu et al. (2005) developed a sharp interface method in order to simulate the impact and spreading of droplets on surfaces with arbitrary shapes. With Level-Set representation of the interfaces and finite difference discretization of the incompressible Navier-Stokes equations, they studied impact of droplets in several scenarios and covered a range of parameters and the obtained results successfully matched other numerical and experimental results. Shen, Bi, and Guo [2012] applied lattice Boltzmann algorithm to simulate the droplet impacting on a $2 \mathrm{D}$ spherical surface. They described the process of droplets impacting on a pipe surface in four stages, which are moving, spreading, nucleating, and dripping and stated that under some certain circumstances, a partial splash occurs. Zhang, Papadikis, and Gu (2014) also employed a 2D lattice Boltzmann method to simulate impact of a liquid droplet onto a curved target and successfully captured three distinct temporal phases as those reported in the experimental study of Bakhshi, Roisman, and Tropea (2007).

Based on a recent and thorough study by Khojasteh et al. (2016b), it is found that a large number of the numerical investigations regarding droplets undergoing collision with SH-surfaces (both planar and non-planar) are reported for non-bouncing cases and numerical modeling of bouncing cases requires further attention. Furthermore, the majority of the numerical studies have been conducted two-dimensionally. Therefore, in 
order to overcome the inadequacies in this area, this study is concerned with 3D numerical simulation of water droplet impact on planar and non-planar H- and SH-surfaces, taking into account the effects of a wide range of parameters, including impact We number, wettability and surface curvature. Validations against available experimental data and models are also provided to verify the solution method and Level-Set method is employed to track the free-surface of the liquid. The results are viewed in a way to elucidate the physics behind the phenomenon. Finally, some of the models provided in literature for flat surfaces are modified to make them suitable for spherical surfaces which can pave the way for more detailed explorations in future.

\section{Governing equations}

In order to obtain the fluid flow field, two equations should be investigated, the first of which is momentum equation, defined as follows for incompressible flow:

$$
\rho \frac{\partial \vec{u}}{\partial t}+\rho(\vec{u} \cdot \nabla) \vec{u}=-\nabla p+\nabla \cdot \mu\left(\nabla \vec{u}+(\nabla \vec{u})^{T}\right)+\sigma \kappa \delta \vec{n}+\mathbf{F}
$$

where $\rho$ is density, $\mu$ is viscosity, $p$ denotes pressure, $\sigma$ is surface tension coefficient, $\delta$ denotes the delta function concentrated at the interface of the two fluids, ${ }^{n}$ is unit normal vector to the interface pointing into the droplets, $\kappa$ is curvature of the fluid-fluid interface, and $\mathbf{F}$ presents external volume forces that are caused by gravitational force. The second equation is continuity which should also be satisfied for the fluid flow:

$$
\frac{\partial \rho_{m}}{\partial t}+\nabla \cdot(\rho \vec{u})=0
$$

For an incompressible fluid, the relation is simplified in the form of: 


$$
\nabla \cdot \vec{u}=0
$$

The momentum and continuity equations are coupled with the Level-Set equation to track the fluid-fluid interface. This equation is defined as Kamali and Dehghan Manshadi (2016):

$$
\frac{\partial \Phi}{\partial t}+\vec{u} \cdot \nabla \Phi=\gamma \nabla \cdot\left[\grave{o} \nabla \Phi-\Phi(1-\Phi) \frac{\nabla \Phi}{|\nabla \Phi|}\right]
$$

In the above equation $\gamma$ and $\epsilon$ are the numerical stabilization parameters such that $\epsilon$ determines the thickness of the interface and $\gamma$ is reinitialization parameter. The righthand side of this equation consists of two terms. The first term is $\epsilon \nabla \phi$ which acts as an artificial diffusivity in order to prevent discontinuities at the interface. The second term is $\phi(1-\phi)(\nabla \phi /|\nabla \phi|)$, which is described as a compressive flux. In the Level-Set equation, $\phi$ is the Level-Set function. This function is zero for the gas phase while for the liquid phase it is equal to one. This function is used to smooth the fluid properties across the interface. For example, density is:

$$
\rho_{m}=\rho_{c}+\left(\rho_{d}-\rho_{c}\right) \Phi
$$

The $\mathrm{c}$ index refers to continues phase and the $\mathrm{d}$ index refers to disperse phase. In terms of Level-Set function, the $\kappa$ in the Navier-Stokes equation can be expressed as:

$$
\kappa=\nabla \cdot \frac{\nabla \Phi}{|\nabla \Phi|}
$$

and ${ }^{n}$ can be specified by (Kamali and Dehghan Manshadi 2016): 


$$
\vec{n}=\frac{\nabla \Phi}{|\nabla \Phi|}
$$

Additionally, the equilibrium C.A is applied as a boundary condition in our simulations which shows acceptable agreement with experimental data in many scientific articles as well as our study as presented in the validation section.

\section{Model definition and validation}

Several cases are simulated in the present study, all of which consist of an initially spherical water droplet surrounded by air, descending toward a solid surface with a specific initial downward velocity, denoted by $\bar{V}$. Figure 1 is an illustration of the initial condition of a sample case studied. In this Figure, $D_{0}$ represents the initial drop diameter, $\bar{V}$ is the impact velocity, and $\bar{g}$ is the gravitational acceleration. The ratio of the solid sphere diameter to the drop diameter $\left(D^{*}\right)$ will be set equal to 2 and 4 in different simulations while the drop's diameter will be held constant and equal to $2.3 \mathrm{~mm}$. The ratio of drop diameter at any instant to its initial diameter $\left(D / D_{0}\right)$ is called normalized diameter in our results.

The physical properties of the materials are given in Table 1 . In this table, $\gamma_{L V}$ represents surface tension between liquid and vapor phases, $\mu_{0}$ is the zero shear viscosity, and $\rho$ is the density of the fluids (Moon, Kim, and Lee 2014). In the first part of the study, isothermal drop impacts at $293.15 \mathrm{~K}$ and $1 \mathrm{~atm}$ are considered, and the mentioned cases are studied at $\mathrm{We}=5$, and then repeated for We numbers of 15 and 30 . In order to investigate the effect of impact on $\mathrm{SH}$ - and $\mathrm{H}$-surfaces, constant equilibrium C.A.s of $163^{\circ}$ and $125^{\circ}$ are applied, respectively. 


\subsection{Numerical validation}

In order to verify the solution method, some of the obtained numerical results are compared to the available experimental data. A comparison of time-lapsed images of water droplet impacting a $\mathrm{SH}$-surface is drawn between the obtained numerical results and the experimental date provided by Wang et al. (2007), as indicated in Figure 2. The equilibrium C.A is $163^{\circ}$ and the velocity of impact is $0.56 \mathrm{~m} / \mathrm{s}$. As is clear from the figure, the spreading, retraction and rebound of the droplet are precisely simulated by the adopted method. Moreover, the normalized diameter versus time is plotted in Figure 3(a) for the mentioned impingement. It is obvious that the normalized diameter decreases monotonically to zero as the droplet contracts and subsequently lifts off the surface. Additionally, we have made a comparison between the simulated normalized diameter of droplet and those of experimental tests represented by Kim et al. (2012), as illustrated in Figure 3(b). In this case, an H-surface with constant equilibrium C.A of $121^{\circ}$ is used and the velocity of impact is $0.5 \mathrm{~m} / \mathrm{s}$. Finally, the results of the simulations are validated against the experimental results of Antonini et al. (2013) for impact of a droplet with diameter of $2.5 \mathrm{~mm}$ and a velocity of $1 \mathrm{~m} / \mathrm{s}$ on surfaces with different C.A.s. The results of these comparisons are also presented in Figure 4. According to Figures. 2-4, it is found that the numerical results are in good agreement with experiments (the maximum deviation is $25 \%$ for only an instant), and can predict the dynamics of droplet impact with high accuracy.

\subsection{Comparing obtained spreading factor to pre-established models}

Moreover, the numerical results of the present study are validated against pre-established correlations for maximum spreading diameter which is an important factor in determining surface wettability. To this end, the ratio between the maximum spreading diameter and 
the initial drop diameter, called spreading factor $\left(D_{\max } / D_{0}\right)$ is analyzed. Numerous models have been proposed in literature which are generally dependent on We and Re numbers. The ones chosen to be compared with the results of the present study are presented in Table 2.

The results of the comparison for impact on the flat and spherical surfaces and for the equilibrium C.A of $125^{\circ}$ and $163^{\circ}$ are presented in Figure 5. It can be noted from the figure that consistency between the results of the current study and the Akao et al. (1980) correlation is exemplary for flat $\mathrm{H}$-surface and for the flat $\mathrm{SH}$-surface, and also the results are in good agreement with Roisman models (2009). For spherical surface, however, the available models are incapable of predicting the spreading diameter accurately, especially for the case with H-surface.

\section{Results and discussion}

\subsection{Effect of equilibrium contact angle}

This section in intended to investigate the effect of the equilibrium C.A between the droplet and the surface on the post-impingement behavior. A comparison of the normalized diameters between surfaces with same curvature but different C.A and at $\mathrm{We}=15$ is presented in Figure 6. This figure indicates that a higher C.A offers a lower normalized diameter and hence, a lower spreading diameter of droplet after impacting the surface. For cases of water droplet impinging spheres, the maximum normalized diameter for C.A of $125^{\circ}$ is 3.3 , while this value for C.A of $163^{\circ}$ is 2.75 .

Figure 7 compares the same effect, this time taking into account the role of surface curvature as well, by presenting the results for flat and spherical surfaces $\left(D^{*}=4\right)$ on the very same diagram. As observed in Figure 7, for droplet impact on flat surfaces, the maximum normalized diameter for C.A of $125^{\circ}$ is 1.68 , while this value for C.A of $163^{\circ}$ 
is 1.39 . This is mainly because small C.A suggests that little energy is needed to cover the surface by drop spreading during the impact, which in turn this low energy requirement can cause a relatively large normalized diameter (Kim et al. 2012; Ukiwe and Kwok 2005).

\subsection{Effect of impact Weber number}

In order to illustrate the effect of We number on the drop-surface interaction, Figure 8 schematically compares the outcomes of impact on a sphere with $\mathrm{D}^{*}=4$ at three We numbers of 5, 15 and 30 , and at a C.A. of $125^{\circ}$. For the impact of droplet on $\mathrm{H}$ - and SHsurfaces and in absence of splashing, the drop can readily spread over the surface and reach its maximum diameter and then, it can retract or stay close to the mentioned maximum spreading. However, the drop's receding is usually followed by a partial or complete rebound through convergence of capillary waves at the apex that entrap bubbles in the drop (Josserand, Sigurdur, and Thoroddsen 2016; Huang et al. 2013).

As is clear from the Figure 8 and for all cases, the drop spreads over the surface until it reaches its maximum diameter. One point that should be taken into consideration is that the time to reach this maximum diameter differs for the investigated cases and as the We number increases the time of reaching maximum spreading diameter decreases. A fundamental feature of a drop impact on a solid substrate is the maximum spreading diameter. The plot of normalized diameter with respect to time with the conditions mentioned above is shown in Figure 9. According to this figure, it is obvious that the spreading diameter increases as result of a rise in We number, which is associated with the kinetic energy of a droplet impinging on the surface. For the lowest We number of 5, the

Maximum normalized diameter is 2.38 , while these maximum values are 3.27 and 4.18 for We numbers of 15 and 30, respectively. Moreover, the time required to reach the 
maximum spread decreases and droplet bounces off the surface sooner with increasing We number.

In addition, the normalized diameters are plotted against time in Figure 10 for impacts on SH-surface. In this figure, a trend similar to that of $\mathrm{H}$-surface is observed for SH-surface with the distinction that normalized diameter reaches its peak in a shorter time and then declines towards zero with a sharper slope than H-surface.

\subsection{Effect of surface curvature}

In this section, the effect of surface curvature on normalized diameter of the droplet impinging on it is studied. To serve this purpose, impacts with We $=15$ are considered and presented for C.A.s of $163^{\circ}$ and $125^{\circ}$, respectively. Figure 11 depicts the image sequence of impact on $\mathrm{SH}$-surfaces with different curvatures from $\mathrm{t}=0$ to $\mathrm{t}=15 \mathrm{~ms}$ with $3 \mathrm{~ms}$ intervals. As observed in the images, for all three cases depicted, the droplet first experiences a deformation into a pancake shape and begins to retract afterwards, and eventually it rebounds off the surface. A point worth noticing is that impact process does not break down the droplet and no splashing or generation of smaller droplets occurs after impact.

Figures 12 and 13 present the normalized diameter of the droplets impinging on SH- and H-surfaces, respectively, and compare the effect of surface curvature for each case at $\mathrm{We}=15$. According to these figures, it can be deduced that the radius of the spherical surface has no tangible effect on the maximum spreading diameter for impact on SH-surface and the spreading diameter remains almost identical for impact on both spheres with $D^{*}=2,4$. The difference between two spherical surfaces is more discernible when impact upon H-surface is considered. However, a change in surface geometry presents a notable difference in contact time of droplet after impinging the surface. The contact time directly reflects the extent of thermal and energy conversions between the 
water droplet and the surface, which is also considered to be crucial to the practical applications (Shen et al. 2014).

To illustrate, consider the case with C.A of $163^{\circ}$ for flat surface and spherical surfaces with $\mathrm{D}^{*}=2,4$ (Figures 11,12 ). It can be readily seen that the processes of water droplets' spreading is followed in a nearly similar manner due to the same initial kinematic parameters of the water droplet in the three cases. However, there is an observable difference in the retracting processes of these cases. As $\mathrm{D}^{*}=2$, the surface delivers a higher hydrophobic performance and the droplet bounces off the surface after $13 \mathrm{~ms}$. The contact times for flat surface and spherical one with $\mathrm{D}^{*}=4$ are $11 \mathrm{~ms}$ and 12 ms, respectively, demonstrating that the drop rebounds off the substrate sooner as the value of $\mathrm{D}^{*}$ increases.

Moreover, for a case with C.A of $125^{\circ}$ and for $\mathrm{D}^{*}=2$, the droplet spreads over the surface but does not rebound off the surface, and remains attached to the surface until droplet's kinetic energy is converted into internal modes of vibration and dissipated through viscosity, while for $\mathrm{D}^{*}=4$, the droplet bounces off the surface after $14 \mathrm{~ms}$. For impact on a flat $\mathrm{H}$-surface, the contact time is similar to that of spherical surface with $\mathrm{D}^{*}=$ 4, as illustrated in Figure 13.

Figure 14 is provided to highlight the effect of surface curvature on the maximum spreading of the droplet over the surfaces. In this figure, the wetted length of the surface measured from the top of the sphere is divided by the corresponding circular chord and plotted against the We number. As shown in the figure, the surface geometry plays an important role in spreading behavior. The smaller the radius, the more it affects the spreading behavior. Therefore, a modification of the constants for all models presented in Table 2 is proposed in this study which offers acceptable consistency with the conducted simulations. For this purpose, a statistical parameter is used similar to that 
employed by Mousavi and Roohi (2013), using a root mean square technique. The value of this parameter ranges from 0 to 1 , with higher values indicating a more accurate numerical solution. These modified models can be used to predict the maximum spreading diameter for impact on spherical surfaces as indicated in Table 3. As is clear from this table, the most accurate modified models are those defined from Akao et al. (1980) and Roisman (2009) with accuracies of 0.92 and 0.88, respectively, and the least accurate is based on Scheller and Bousfield's (1995) model with an accuracy of 0.80.

As stated by Wang et al. $(2008 ; 2011)$, one effect that must be considered in studying droplet impact is gravity. This is normally represented by Bond number (Bo) which is defined as the ratio between gravity force and surface tension force. With the definition of $B o=\frac{\rho g D^{2}}{\gamma}$, the Bo number is equal to 0.72 in the present study which has a relatively important effect, particularly with regard to droplet spreading behavior on curved surfaces. In order to examine this effect, the case of impact on an $\mathrm{H}$ spherical surface with $\mathrm{D}^{*}=2$ and $\mathrm{We}=15$ is repeated, this time without the presence of gravity in the simulation. Results show that gravity indeed plays a role in spreading behavior of droplets on curved surfaces as indicated in Figure 15. As can be observed in the figure, at some time steps, the normalized diameter can deviate up to $17 \%$ between the cases with and without the presence of gravity. Furthermore, with regard to contact time, the figure indicates that after $15 \mathrm{~ms}$, the case of impact without the gravity leads to a complete detachment of droplet from the solid surface, contrary to the other case.

Another force, other than gravity, whose presence causes dramatic differences in spreading behavior between curved and flat surfaces is centrifugal force. In fact, a parameter $(\xi)$ which is defined to indicate the relative importance of gravity $\left(F_{g}\right)$ and centrifugal force $\left(F_{c}\right)$ shows that centrifugal force is for most of the time higher than the 
gravity force. The definition is derived from the forces acting on a generic volume of liquid at the rim of the lamella, leading to following formula:

$$
\xi=\frac{F_{c}}{F_{g}}=\frac{V_{\text {spread }}^{2}}{g R \cdot \tan \left(\frac{l}{R}\right)}
$$

Where $l$ is the semi-length of the spreading and $V_{\text {spread }}$ is the spreading velocity.

Results presented in Figure 16 demonstrate that the centrifugal force is indeed of a higher level of influence than gravity. According to this figure, it is clear that the spreading ratio on a curved surface is higher than for a flat one. The main reason is due to the centrifugal force rather than the gravity force. Both vectors are contributing to the extension of the lamella, but the first force is dominating almost until the end of the spreading process.

\section{Conclusions}

Investigating the underlying dynamics of droplet undergoing collision with solid substrates, which is an active area of research, has attracted a great deal of attention among scholars and technicians. Therefore, different from previous research, in which attention was mainly focused on the 2D modeling of non-bouncing droplets, a detailed study on the impact and rebound dynamics of a drop on curved and flat $\mathrm{H}$ - and $\mathrm{SH}-$ surfaces was presented, considering the effects of surface curvature, C.A, and We numbers. It was found that the predicted spreading factor for impact on flat surfaces is in good agreement with the available models, while these models are incapable of offering accurate spreading factor for curved surfaces. Considering the effect of substrates, the diameter of the spherical surface has insignificant effect on the spreading diameter, and the contact time of droplet is almost same for flat surface and for sphere surface with a higher value of $\mathrm{D}^{*}$. The droplet undergoes a deformation into a pancake shape and begins to retract afterwards, and eventually it rebounds off the surface in all cases except for an 
H-surface when $\mathrm{D}^{*}=2$. Additionally, the spreading diameter increases as the C.A decreases, due to the fact that little energy is required to cover the surface by drop spreading during the impact. The maximum spreading diameter and the time needed to reach this maximum spread decrease with an increase in We number, providing a higher kinetic energy to impinging droplet. Moreover, a modification of the constants for the available models that predict the spreading factor of droplet impacting flat surfaces was carried out to make them capable of predicting the spreading factor for impingement on spherical surfaces. Finally, the analysis of the gravity force and centrifugal force revealed that it is the latter one which is allowing a higher spreading ratio on curved surfaces than flat ones.

\section{References}

Aboud, D. G. and A.-M. Kietzig. 2015. "Splashing threshold of oblique droplet impacts on surfaces of various wettability." Langmuir 31(36):10100-10111.

Akao, F., K. Araki, S. Mori, and A. Moriyama. 1980. "Deformation behaviours of a liquid droplet impinging onto hot metal surface.” Trans. Iron Steel Inst. Jpn. 20(11):737-743.

Antonini, C., A. Amirfazli, and M. Marengo. 2012. "Drop impact and wettability: From hydrophilic to superhydrophobic surfaces." Physics of fluids 24(10):102104.

Antonini, C., F. Villa, I. Bernagozzi, A. Amirfazli, and M. Marengo. 2013. "Drop rebound after impact: The role of the receding contact angle." Langmuir 29(52):1604516050.

Bakshi, S., I. V. Roisman, and C. Tropea. 2007. "Investigations on the impact of a drop onto a small spherical target." Physics of fluids 19(3):032102.

Bange, P. G. and R. Bhardwaj. 2016. "Computational study of bouncing and nonbouncing droplets impacting on superhydrophobic surfaces." Theoretical and Computational Fluid Dynamics 30(3):211-235.

Binesh, A., S. Mousavi, and R. Kamali. 2015. "Effect of temperature-dependency of Newtonian and non-Newtonian fluid properties on the dynamics of droplet impinging on 
hot surfaces.” International Journal of Modern Physics C 26(09):1550106.

Clanet, C., C. Béguin, D. Richard, and D. Quéré. 2004. "Maximal deformation of an impacting drop.” Journal of Fluid Mechanics 517:199-208.

Dehghan Manshadi, M. K., D. Khojasteh, M. Mohammadi, and R. Kamali. 2016. "Electroosmotic micropump for lab on a chip biomedical applications." International Journal of Numerical Modelling: Electronic Networks, Devices and Fields 29(5):845858.

Hardalupas, Y., A. Taylor, and J. Wilkins. 1999. "Experimental investigation of submillimetre droplet impingement on to spherical surfaces." International Journal of Heat and Fluid Flow 20(5):477-485.

Hartley, G. and R. Brunskill. 1958. "Reflection of water drops from surfaces." Surface phenomena in chemistry and biology 214.

Hung, L. and S. Yao. 1999. "Experimental investigation of the impaction of water droplets on cylindrical objects." International journal of multiphase flow 25(8):15451559.

Hung, Y.-L., M.-J. Wang, J.-W. Huang, and S.-Y. Lin. 2013. "A study on the impact velocity and drop size for the occurrence of entrapped air bubbles-Water on parafilm." Experimental Thermal and Fluid Science 48:102-109.

Josserand, C. and S. T. Thoroddsen. 2016. "Drop impact on a solid surface." Annual review of fluid mechanics 48:365-391.

Kamali, R., D. Khojasteh, and S. M. Mousavi. 2016. "Newtonian and non-Newtonian Droplet Impact onto a Heated Hydrophobic Solid Surface." Paper presented at the annual meeting for 24th Annual International Conference on Mechanical Engineering-ISME, Yazd, Iran, April 26-28.

Kamali, R. and M. K. D. Manshadi. 2016. "Numerical simulation of the leaky dielectric microdroplet generation in electric fields." International Journal of Modern Physics $C$ 27(01):1650012.

Khojasteh, D., M. K. Dehghan Manshadi, S. M. Mousavi, and R. Kamali. 2016a. "Droplet impact on superhydrophobic surface under the influence of an electric field." Paper presented at the annual meeting for 3rd Annual International Conference on New Research Achievements in Chemistry \& Chemical Engineering, Tehran, Iran, September. 
Khojasteh, D., M. Kazerooni, S. Salarian, and R. Kamali. 2016b. "Droplet impact on superhydrophobic surfaces: A review of recent developments." Journal of Industrial and Engineering Chemistry 42:1-14.

Khojasteh, D., S. M. Mousavi, and R. Kamali. 2017. "CFD analysis of Newtonian and non-Newtonian droplets impinging on heated hydrophilic and hydrophobic surfaces." Indian Journal of Physics 91(5):513-520.

Kim, H., C. Lee, M. H. Kim, and J. Kim. 2012. "Drop impact characteristics and structure effects of hydrophobic surfaces with micro-and/or nanoscaled structures." Langmuir 28(30):11250-11257.

Kim, J. 2007. "Spray cooling heat transfer: the state of the art." International Journal of Heat and Fluid Flow 28(4):753-767.

Liu, H., S. Krishnan, S. Marella, and H. Udaykumar. 2005. "Sharp interface Cartesian grid method II: A technique for simulating droplet interactions with surfaces of arbitrary shape.” Journal of Computational Physics 210(1):32-54.

Liu, Y., G. Whyman, E. Bormashenko, C. Hao, and Z. Wang. 2015. “Controlling drop bouncing using surfaces with gradient features." Applied Physics Letters 107(5):051604.

Liu, Y. C., T. Farouk, A. J. Savas, F. L. Dryer, and C. T. Avedisian. 2013. "On the spherically symmetrical combustion of methyl decanoate droplets and comparisons with detailed numerical modeling." Combustion and Flame 160(3):641-655.

Manshadi, M. K. D., D. Khojasteh, A. Mansoorifar, and R. Kamali. 2016. "Efficiency enhancement of ICEK micromixer by a rectangular obstacle." Paper presented at the annual meeting for 3rd Annual International Conference on New Research Achievements in Chemistry \& Chemical Engineering, Tehran, Iran, September.

Moon, J. H., D. Y. Kim, and S. H. Lee. 2014. "Spreading and receding characteristics of a non-Newtonian droplet impinging on a heated surface." Experimental Thermal and Fluid Science 57:94-101.

Mousavi, S. M. and E. Roohi. 2014. "Large eddy simulation of shock train in a convergent-divergent nozzle." International Journal of Modern Physics $C$ 25(04):1450003.

Pasandideh-Fard, M., M. Bussmann, and S. Chandra. 2001. "Simulating droplet impact on a substrate of arbitrary shape." Atomization and sprays 11(4). 
Roisman, I. V. 2009. "Inertia dominated drop collisions. II. An analytical solution of the Navier-Stokes equations for a spreading viscous film.” Physics of fluids 21(5):052104.

Scheller, B. L. and D. W. Bousfield. 1995. "Newtonian drop impact with a solid surface." AIChE Journal 41(6):1357-1367.

Shen, S., F. Bi, and Y. Guo. 2012. "Simulation of droplets impact on curved surfaces with lattice Boltzmann method." International Journal of Heat and Mass Transfer 55(23):6938-6943.

Shen, Y., Tao, J., Tao, H., Chen, S., Pan, L. and Wang, T., 2015. "Relationship between wetting hysteresis and contact time of a bouncing droplet on hydrophobic surfaces." ACS applied materials \& interfaces 7(37):20972-20978.

Tsai, P., S. Pacheco, C. Pirat, L. Lefferts, and D. Lohse. 2009. "Drop impact upon microand nanostructured superhydrophobic surfaces.” Langmuir 25(20):12293-12298.

Ukiwe, C. and D. Y. Kwok. 2005. "On the maximum spreading diameter of impacting droplets on well-prepared solid surfaces.” Langmuir 21(2):666-673.

Wang, F. C., Feng, J. T., and Zhao, Y. P. 2008. "The head-on colliding process of binary liquid droplets at low velocity: High-speed photography experiments and modeling." Journal of colloid and interface science, 326(1):196-200.

Wang, F.C., Yang, F. and Zhao, Y.P., 2011. "Size effect on the coalescence-induced selfpropelled droplet." Applied Physics Letters, 98(5):053112.

Wang, Z., C. Lopez, A. Hirsa, and N. Koratkar. 2007. "Impact dynamics and rebound of water droplets on superhydrophobic carbon nanotube arrays." Applied Physics Letters 91(2):023105.

Xu, K. and J. R. Heath. 2013. "Wetting: Contact with what?" Nature materials 12(10):872-873.

Yarin, A. 2006. "Drop impact dynamics: splashing, spreading, receding, bouncing...." Annu. Rev. Fluid Mech. 38:159-192.

Yusof, A., H. Keegan, C. D. Spillane, O. M. Sheils, C. M. Martin, J. J. O'Leary, R. Zengerle, and P. Koltay. 2011. "Inkjet-like printing of single-cells." Lab on a Chip 11(14):2447-2454.

Zhang, D., K. Papadikis, and S. Gu. 2014. "Investigations on the droplet impact onto a 
spherical surface with a high density ratio multi-relaxation time lattice-Boltzmann model." Communications in Computational Physics 16(04):892-912. 

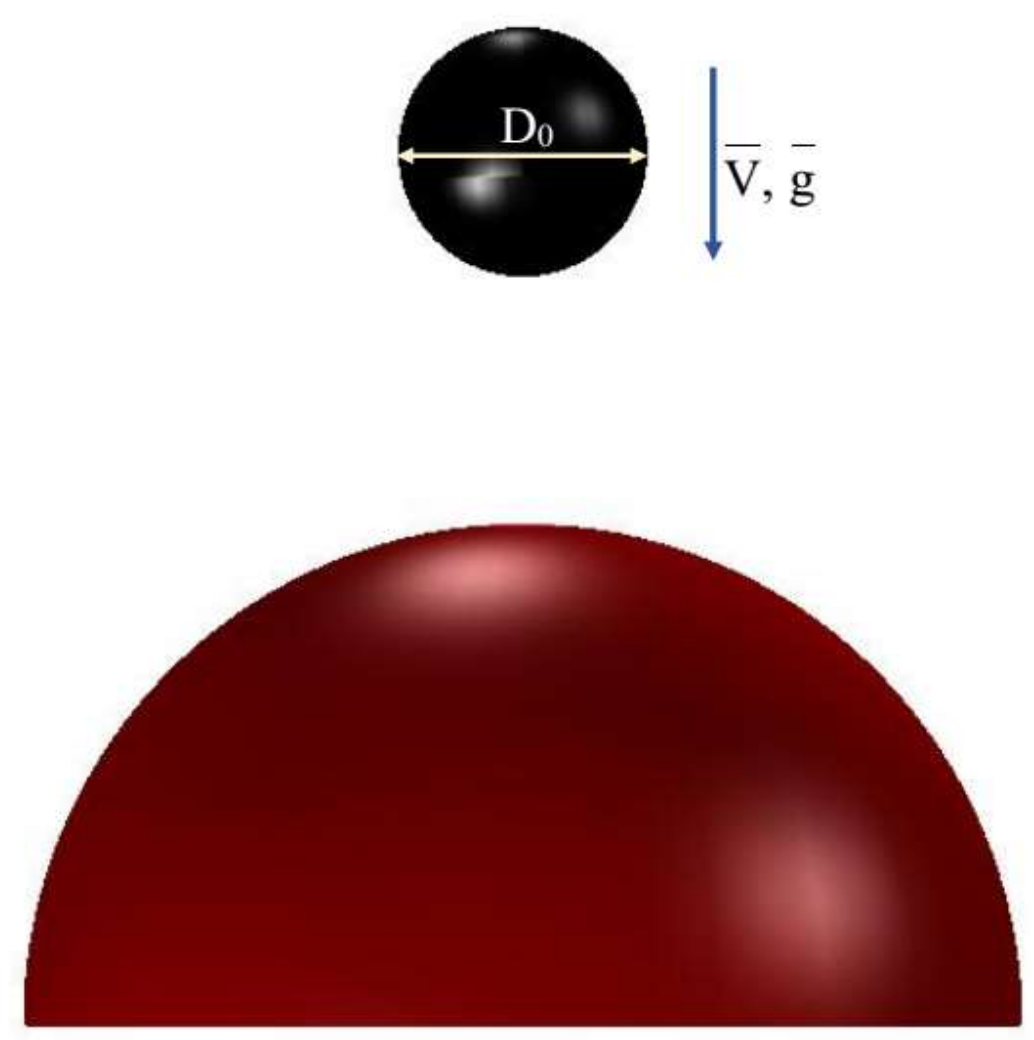

Figure 1. Initial conditions in a sample case. 
(a)

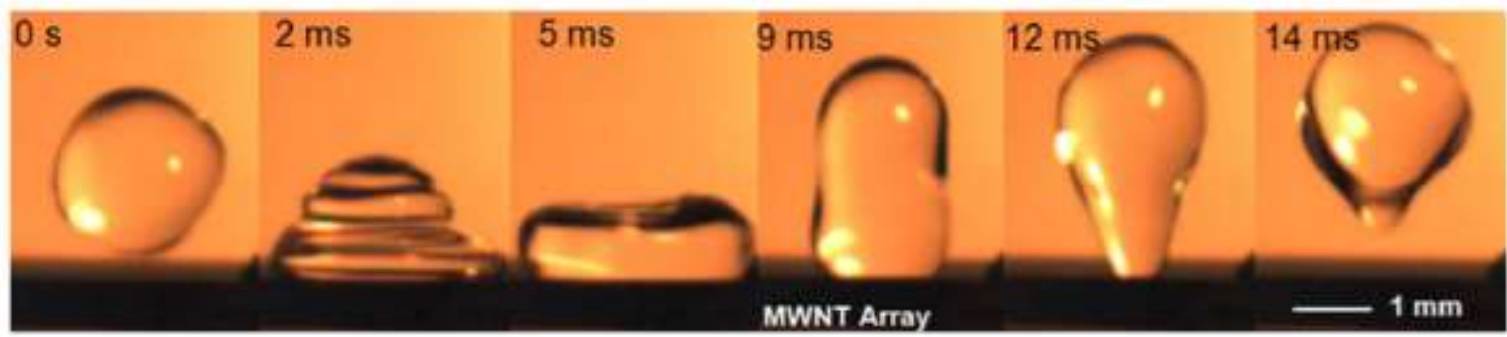

(b)

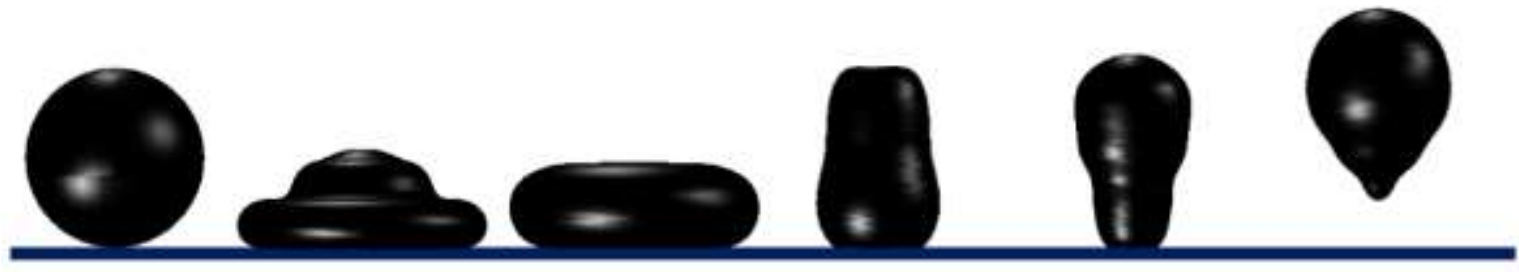

Figure 2. A comparison between: (a) experimental; and (b) numerical time-lapsed snapshots of water droplet impinging a SH-surface. 


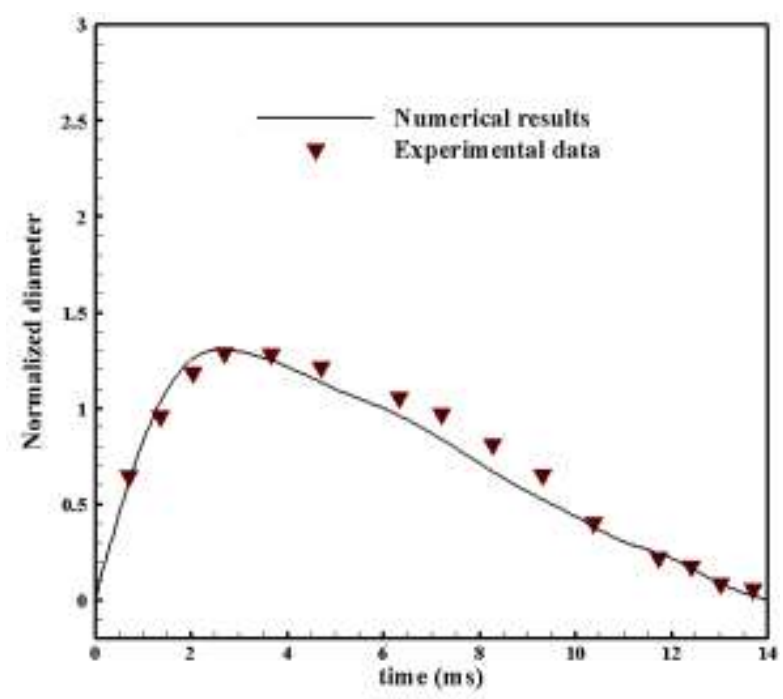

(a)

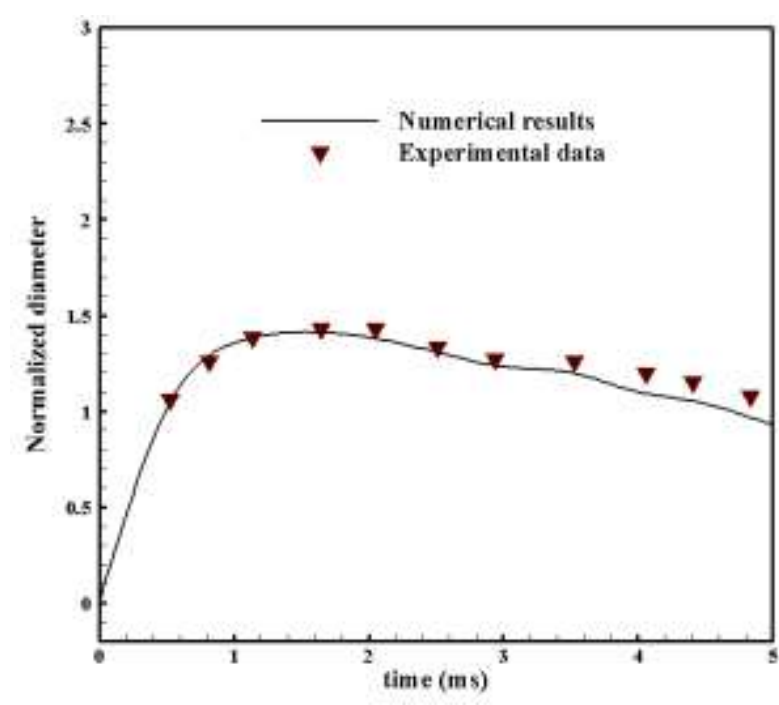

(b)

Figure 3. Comparison between numerical results of the current study and experimental results of: (a) Wang et al. (2007); and (b) Kim et al. (2012). 


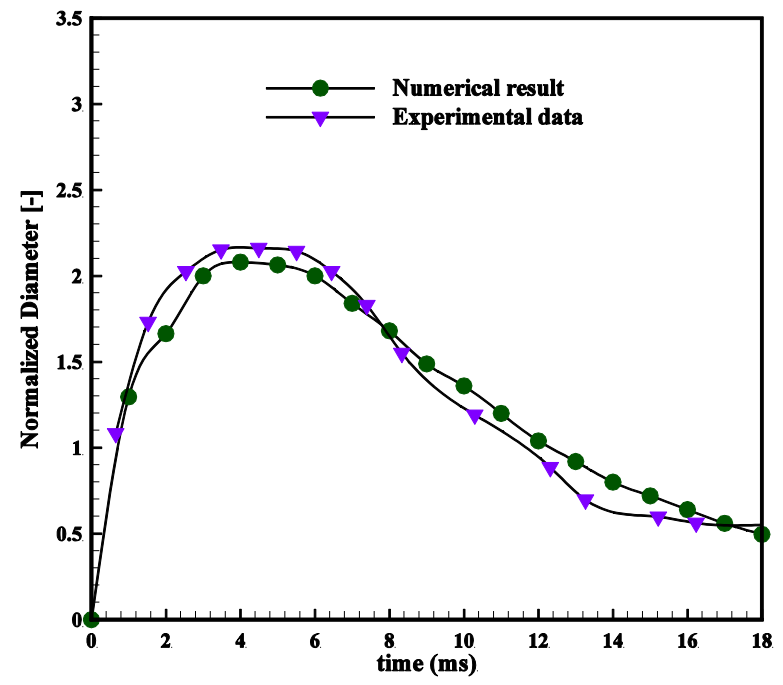

(a)

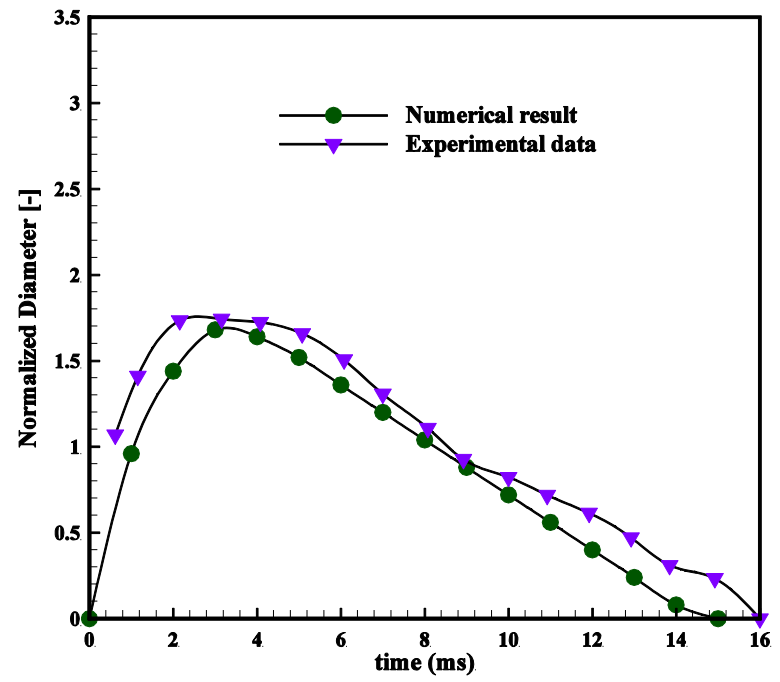

(b)

Figure 4. Comparison between numerical results of the current study and experimental results of Antonini et al. [15] for C.A.s of: (a) $91^{\circ}$; and (b) $145^{\circ}$. 


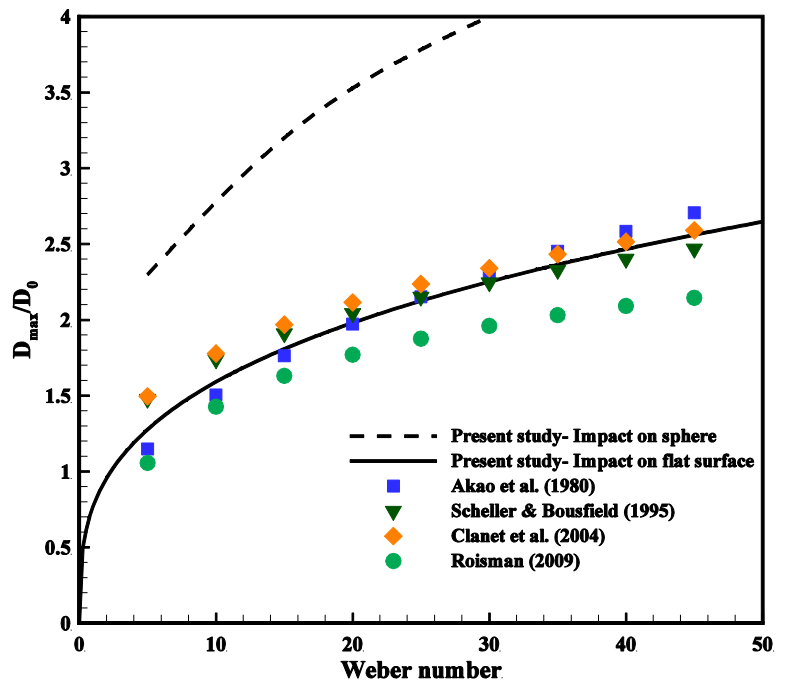

(a)

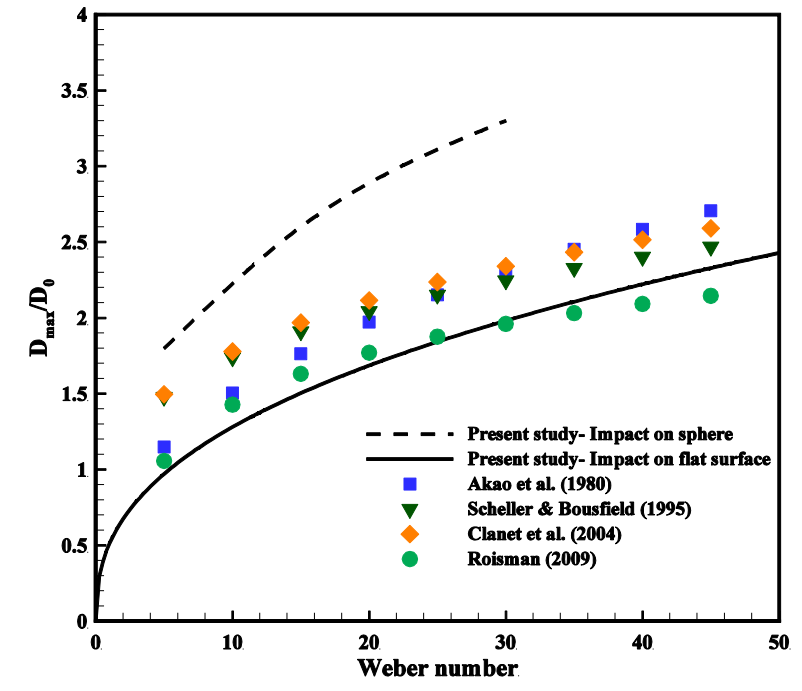

(b)

Figure 5. Comparison of obtained spreading factor to pre-established models for C.A.s

$$
\text { of: (a) } 125^{\circ} \text {; and (b) } 163^{\circ} \text {. }
$$




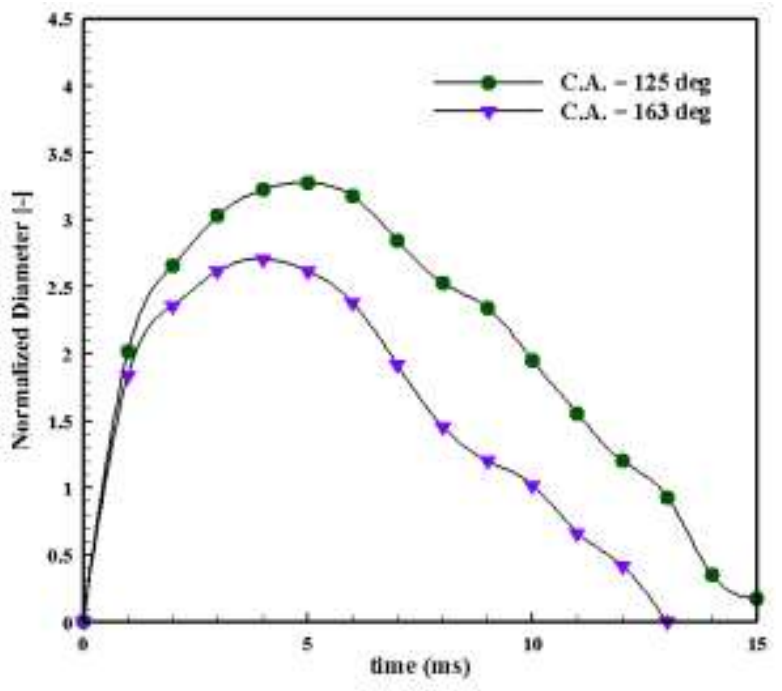

(a) $\mathrm{D}^{*}=2$

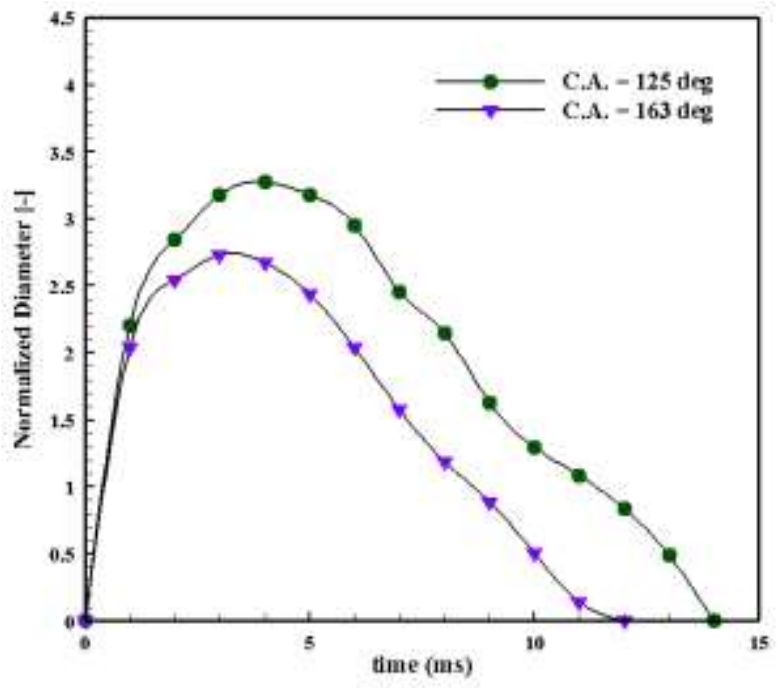

(b) $\mathrm{D}^{*}=4$

Figure 6. Normalized diameter with respect to time at different C.A.s and at $\mathrm{We}=15$ when: (a) $D^{*}=2$; and (b) $D^{*}=4$. 


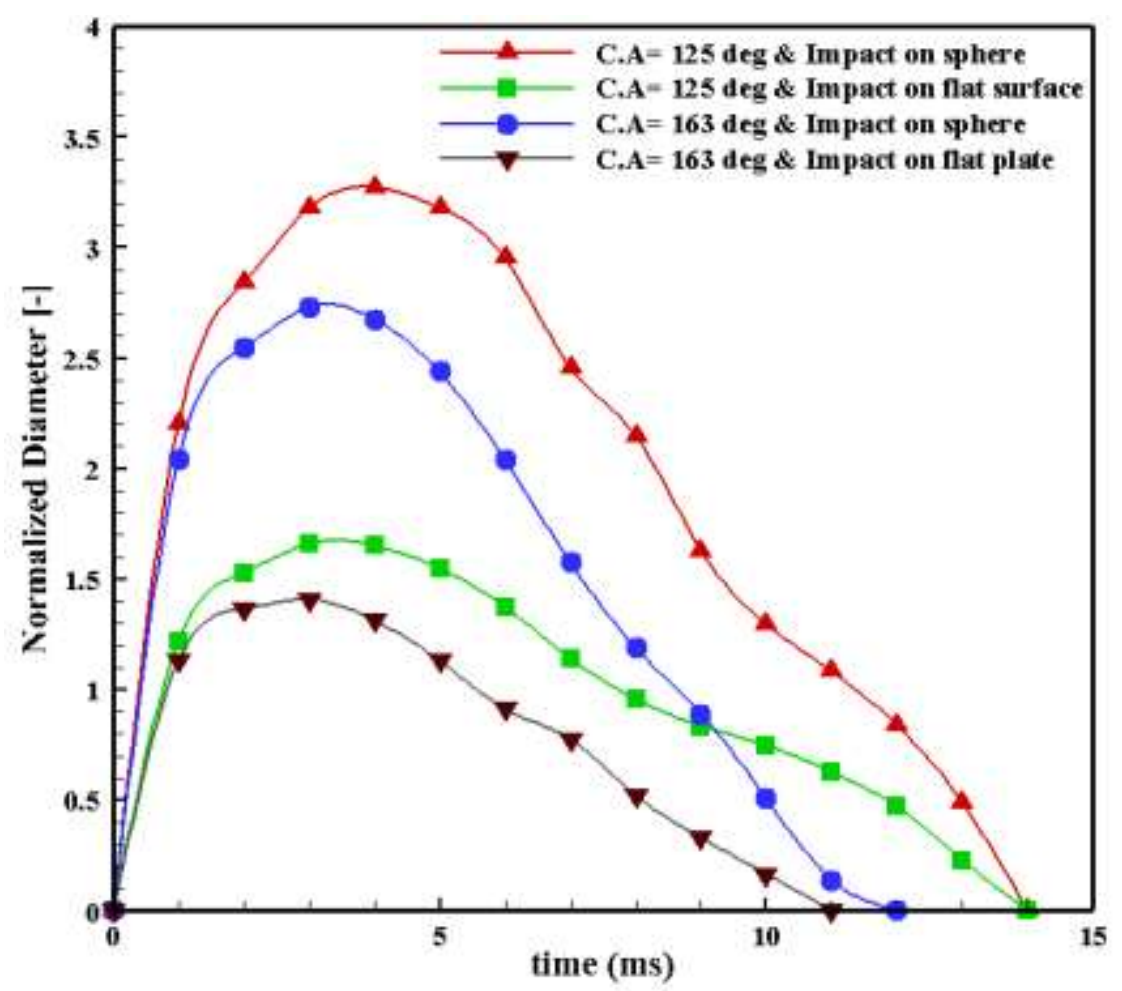

Figure 7. Normalized diameter of droplets impacting on surfaces versus time for different surfaces and C.A.s. 


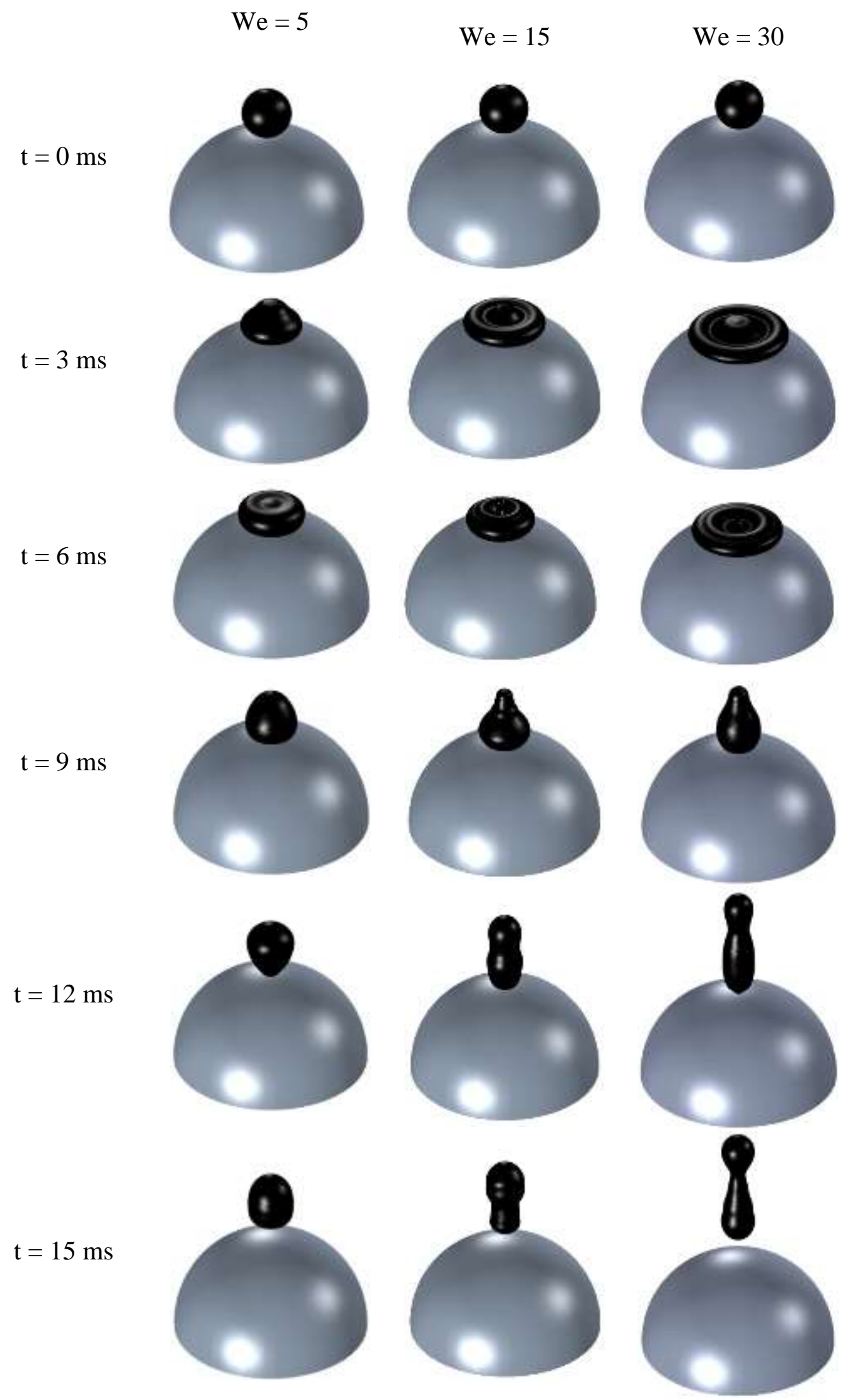

Figure 8. Time evolution of droplet shape impacting on the sphere when $\mathrm{D}^{*}=4$ and C.A of $125^{\circ}$. 


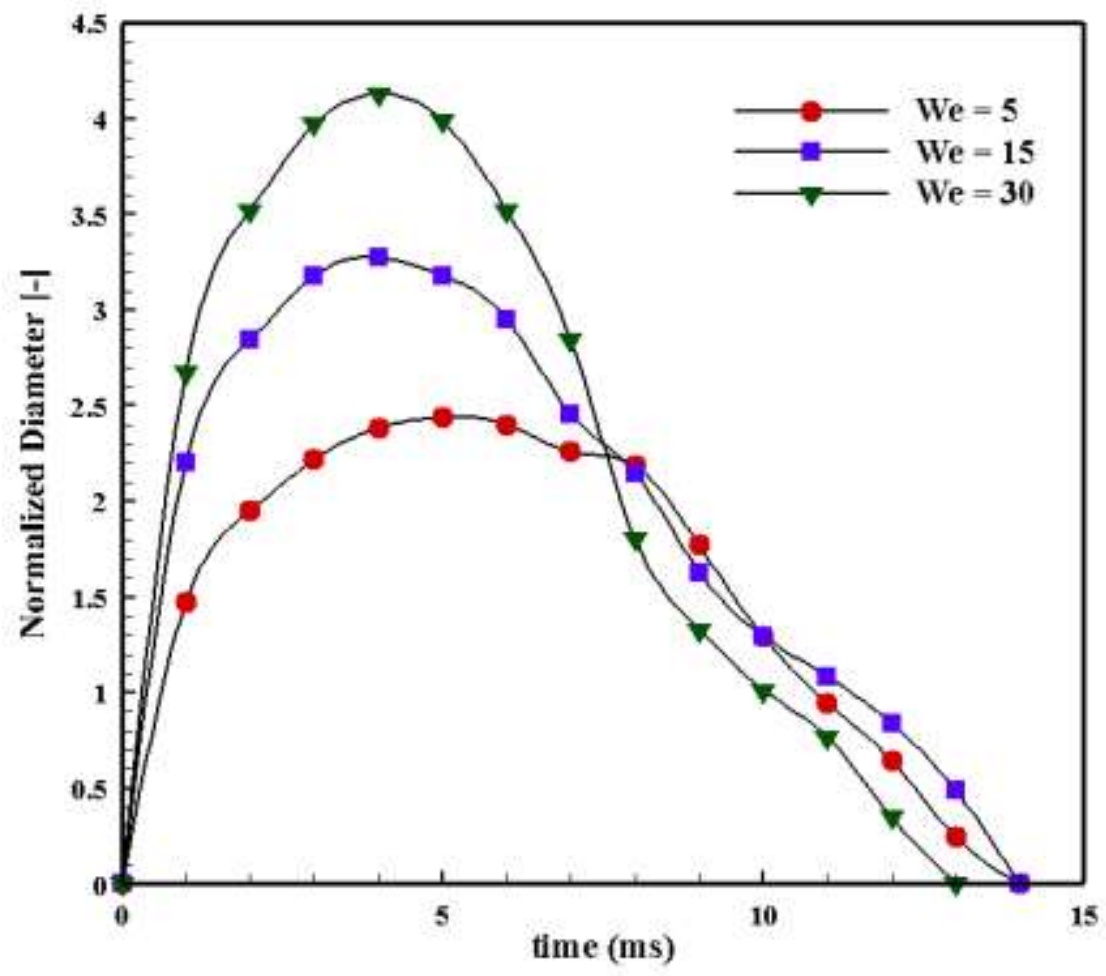

Figure 9. Normalized diameter of droplets impacting on an $\mathrm{H}$-surface versus time when $\mathrm{D}^{*}=4$ and at three different We numbers. 


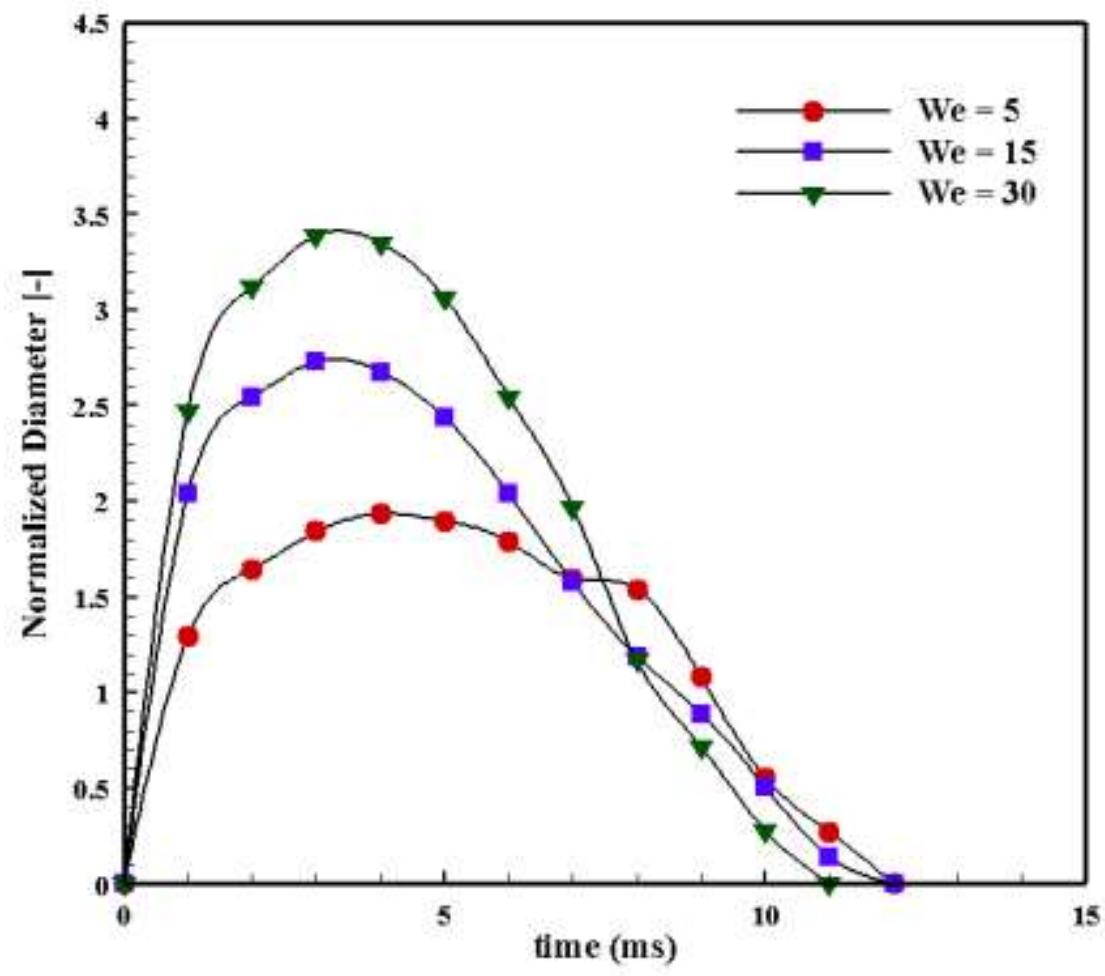

Figure 10. Normalized diameter of droplets impacting on a SH-surface versus time when $\mathrm{D}^{*}=4$ and at three different We numbers. 


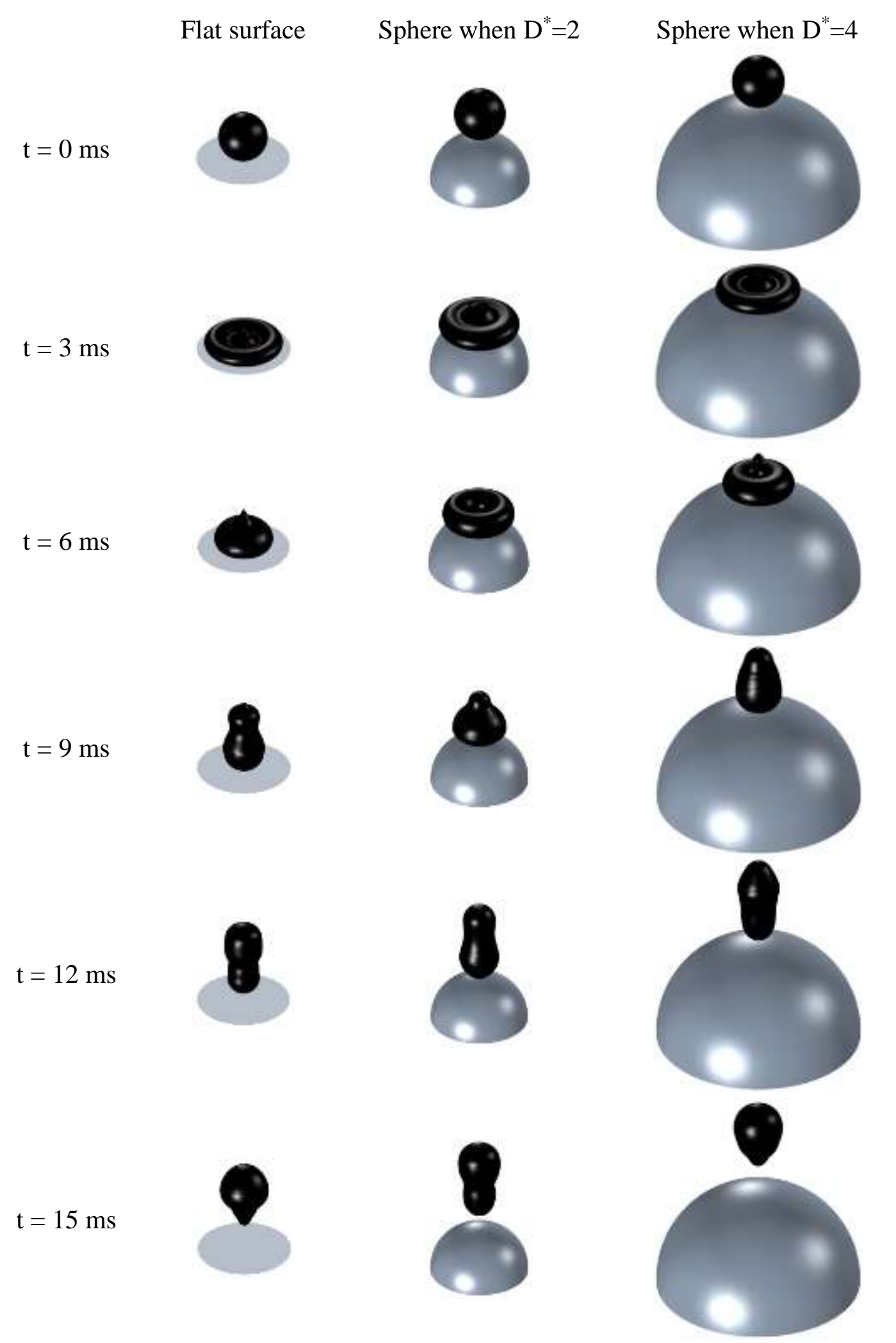

Figure 11. Time evolution of droplet shape impacting different surfaces with C.A of $163^{\circ}$ and for $\mathrm{We}=15$. 


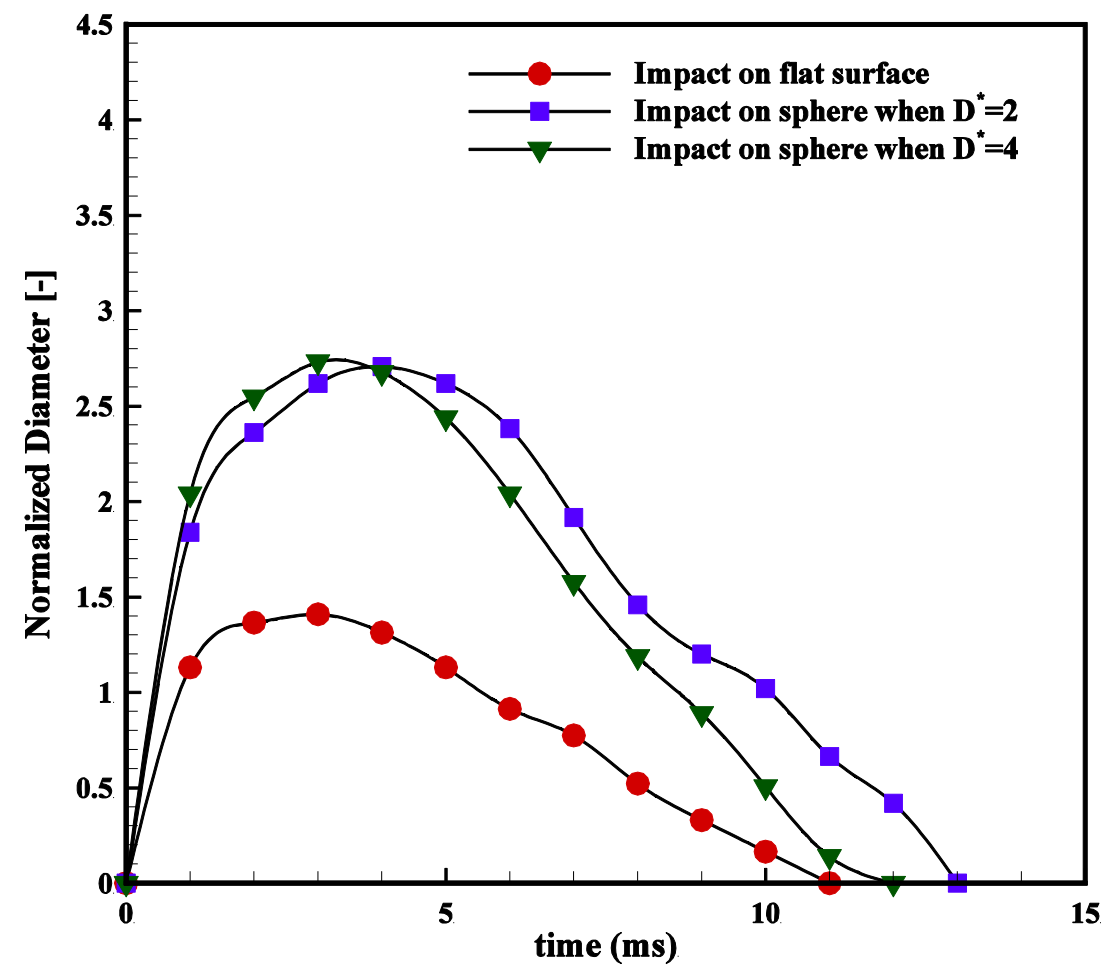

Figure 12. Normalized diameter of the droplet impinging on a SH-surface with respect to time for $\mathrm{We}=15$. 


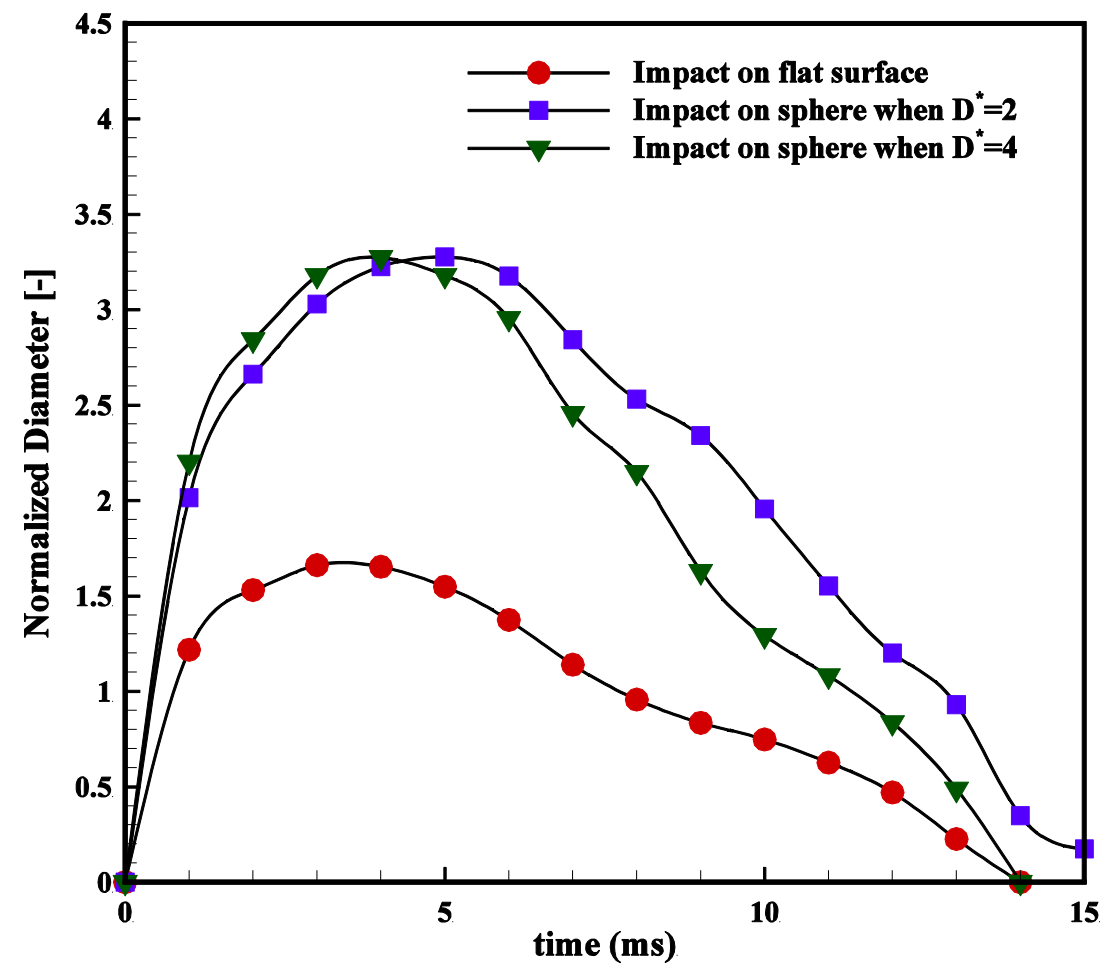

Figure 13. Normalized diameter of the droplet impinging on an H-surface with respect to time for $\mathrm{We}=15$. 


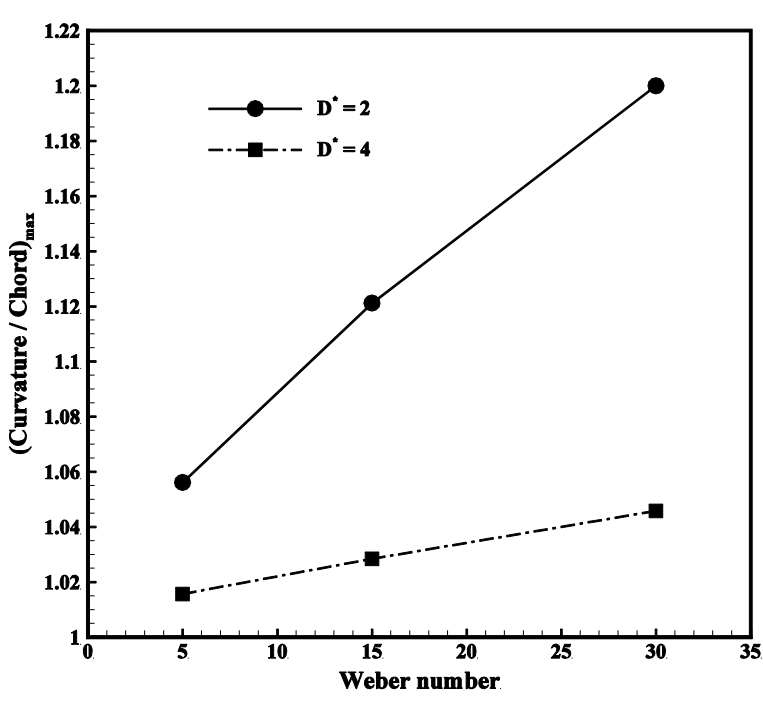

(a)

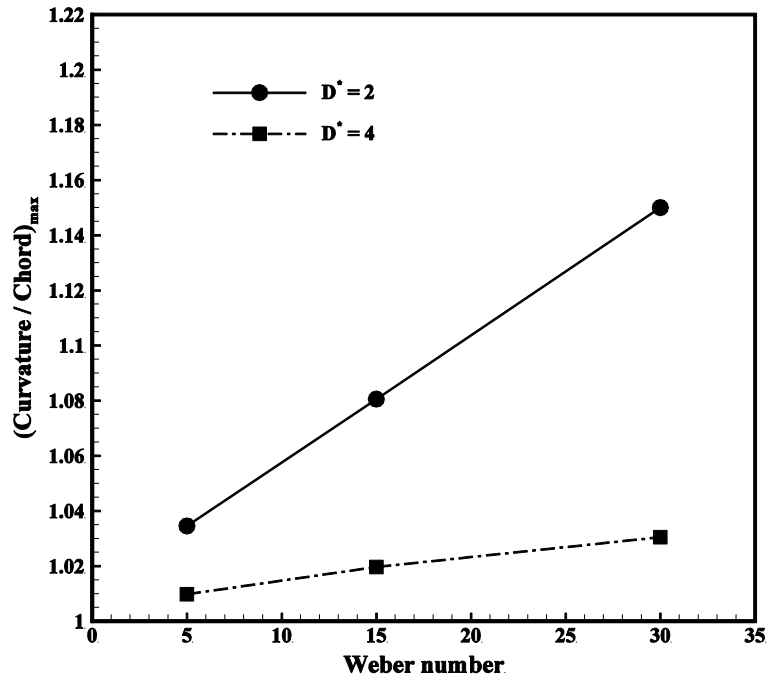

(b)

Figure 14. Ratio of wetted curved length along the surface to the corresponding circular chord as a function of We number for C.A.s of: (a) $125^{\circ}$; and (b) $163^{\circ}$. 


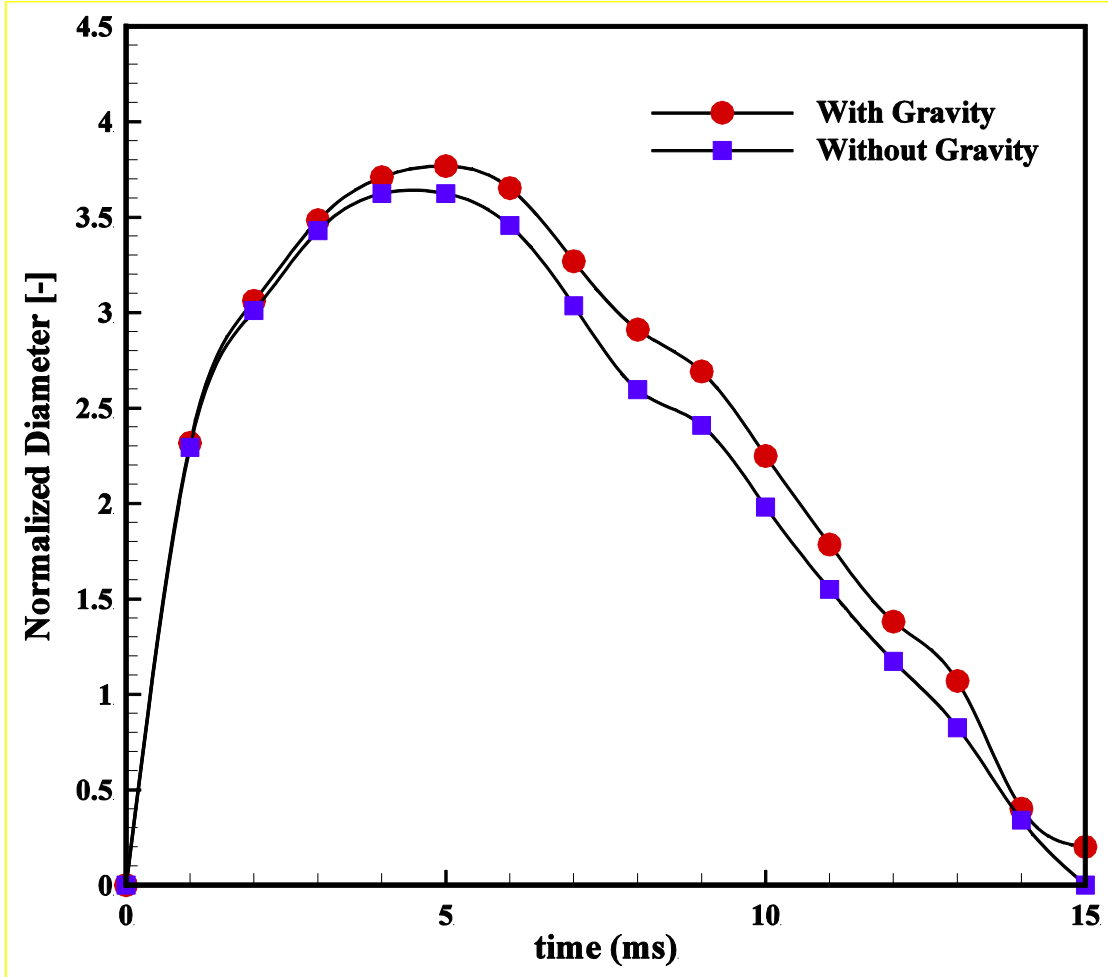

Figure 15. Effect of gravity on droplet impact onto an $\mathrm{H}$ spherical surface with $\mathrm{D}^{*}=2$ and $\mathrm{We}=15$. 


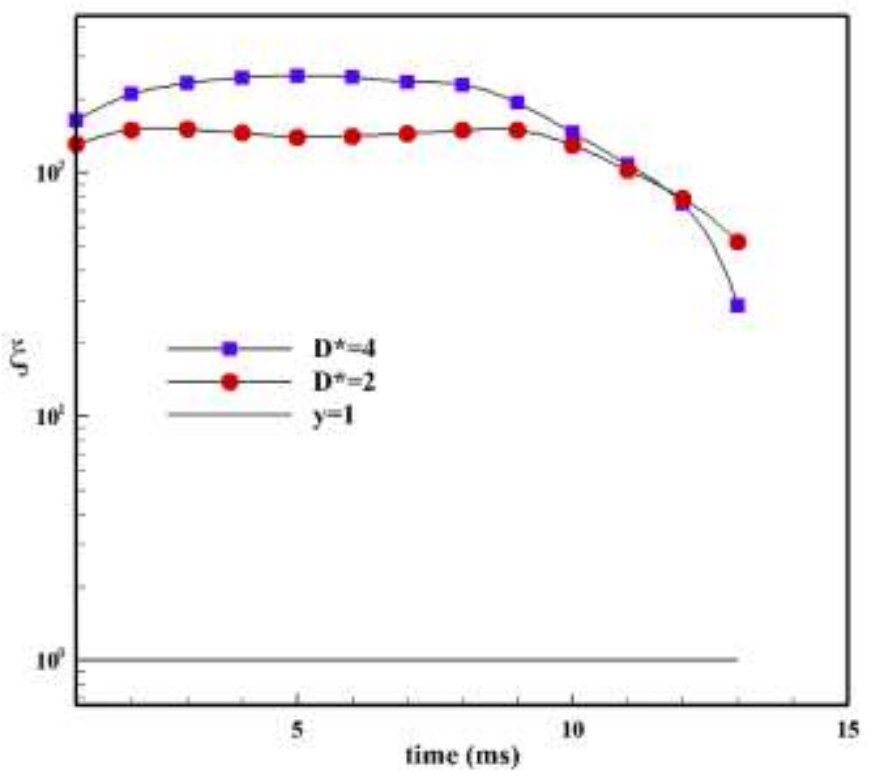

(a)

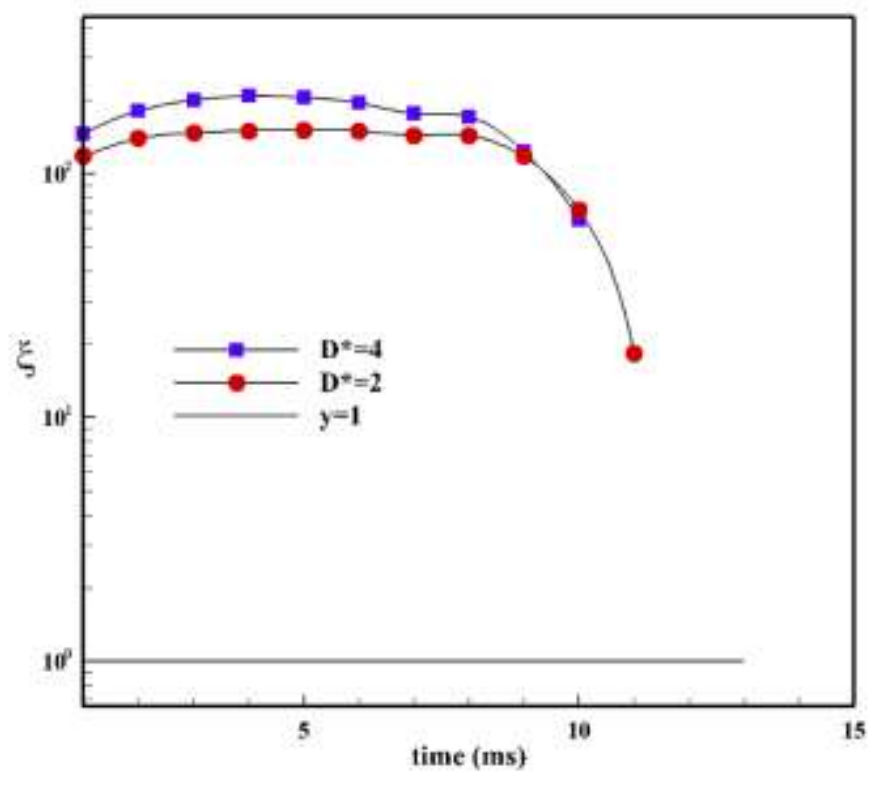

(b)

Figure 16. Ratio of centrifugal force to gravity force at C.A.s of: (a) $125^{\circ}$; and (b) $163^{\circ}$. 
Table 1. Physical properties of water droplet (Moon et al., 2014).

\begin{tabular}{|c|c|c|c|}
\hline Material & $\mu_{0}$ (Pa.s) & $\rho\left(\mathrm{kg} / \mathrm{m}^{3}\right)$ & $\gamma_{L V}(\mathrm{~N} / \mathrm{m})$ \\
\hline Water droplet & 0.0089 & 998 & 0.072 \\
\hline
\end{tabular}

Table 2. The models used for validation and their expressions

\begin{tabular}{|c|c|}
\hline Model & Spreading factor $\left(\mathrm{D}_{\max } / \mathrm{D}_{0}\right):$ \\
\hline Akao et al. (1980) & $0.613 W e^{0.39}$ \\
\hline Scheller and Bousfield (1995) & $0.61 R e^{1 / 5}\left(W e R e^{-2 / 5}\right)^{1 / 6}$ \\
\hline Clanet et al. (2004) & $W e^{1 / 4}$ \\
\hline Roisman (2009) & $0.87 R e^{1 / 5}-0.4 R e^{2 / 5} W e^{-1 / 2}$ \\
\hline
\end{tabular}


Table 3. Modified models to predict spreading factor of impact on spherical surfaces.

\begin{tabular}{|c|c|c|c|}
\hline Model & $\begin{array}{c}\text { Spreading factor }\left(\mathrm{D}_{\max } / \mathrm{D}_{0}\right) \\
-\end{array}$ & $\begin{array}{c}\text { Spreading factor }\left(\mathrm{D}_{\max } / \mathrm{D}_{0}\right) \\
-\end{array}$ & Estimation error \\
& flat surfaces & $W e^{0.39}$ & 0.92 \\
\hline $\begin{array}{c}\text { Akao et al. (1980) } \\
\text { Scheller and } \\
\text { Bousfield (1995) }\end{array}$ & $0.61 R e^{1 / 5}\left(W e R e^{-2 / 5}\right)^{1 / 6}$ & $0.95 R e^{1 / 5}\left(W e R e^{-2 / 5}\right)^{1 / 6}$ & 0.80 \\
\hline $\begin{array}{c}\text { Clanet et al. } \\
(2004)\end{array}$ & $W e^{1 / 4}$ & $1.5 W e^{1 / 4}$ & 0.83 \\
\hline $\begin{array}{c}\text { Roisman (2009) } \\
\text { curfaces }\end{array}$ & $-0.4 R e^{2 / 5} W e^{-1 / 2}$ & $\begin{array}{l}1.5 R e^{1 / 5} \\
-0.6 R e^{2 / 5} W e^{-1 / 2}\end{array}$ & 0.88 \\
\hline
\end{tabular}

